\title{
Effects of temperature on aging degradation of soft and hard lead zirconate titanate ceramics
}

Methee Promsawat ${ }^{1}$, Asawin Phuetthonglang ${ }^{2}$, Boonruang Marungsri ${ }^{2}$, Napatporn Promsawat ${ }^{3}$, Pattanaphong Janphuang ${ }^{4}$, Zhenhua Luo ${ }^{5}$ and Soodkhet Pojprapai ${ }^{3,6, *}$

${ }^{1}$ Department of Materials Science and Technology, Faculty of Science, Prince of Songkla University, Songkhla 90112, Thailand

${ }^{2}$ School of Electrical Engineering, Institute of Engineering, Suranaree University of Technology, Nakhon Ratchasima 30000, Thailand

${ }^{3}$ School of Ceramic Engineering, Institute of Engineering, Suranaree University of Technology, Nakhon Ratchasima 30000, Thailand

${ }^{4}$ Synchrotron Light Research Institute, Nakhon Ratchasima 30000, Thailand

${ }^{5}$ School of Water, Energy and Environment, Cranfield University, Cranfield MK43 OAL, UK

${ }^{6}$ Data Storage Science and Technology Research Unit, Suranaree University of Technology, Nakhon Ratchasima 30000, Thailand

*Corresponding author. Tel.: +66 44 224542; fax: +66 44224477

E-mail address: soodkhet@g.sut.ac.th (S. Pojprapai)

\begin{abstract}
This paper aims to study the effects of heat treatment temperatures on the aging degradation of piezoelectric properties, i.e. piezoelectric coefficient $\left(d_{33}\right)$ and planar electromechanical coupling factor $\left(k_{p}\right)$, in soft and hard PZT ceramics. Aging degradations of $d_{33}$ and $k_{p}$ of the samples were measured for 192 hours prior to heat treatments. The samples were then treated at various temperatures equivalent to $0.3,0.4,0.5,0.6,0.7$ and 0.8 times of
\end{abstract}


the materials' Curie temperatures. Aging degradations of $d_{33}$ and $k_{p}$ of heat-treated samples were observed continuously for 1128 hours. The piezoelectric properties of un-treated samples gradually decreased with aging time. Attenuation of $d_{33}$ and $k_{p}$ in the samples immediately after heat treatment increased with increasing heat treatment temperature. Moreover, aging degradation rate and relaxation time measured after heat treatments increased with increasing heat treatment temperature. Comparing to hard PZT ceramics, soft PZT demonstrated greater change of $d_{33}$ and $k_{p}$ immediately after heat treatments. Soft PZT also showed greater aging rate and aging time than those of hard PZT. From the overall results, it can be concluded that both material type and heat treatment temperature have effects on aging behaviors of PZT materials. Aging degradation was more pronounced in soft PZT and the samples treated at high temperatures. The observed aging behaviors of PZT materials were explained by interaction between domains and oxygen vacancies acting as defects leading to volume, domain and grain boundary effects.

Keywords: aging; heat treatment; piezoelectric properties; PZT

\section{Introduction}

Lead zirconate titanate (PZT) is a piezoelectric ceramic with excellent electromechanical properties. It is commonly used in the applications of piezo-transducers, actuators and sensors [1-4], occupying more than $90 \%$ of the bulk piezoelectric device market [5]. PZT ceramics are usually glued to other electronic parts using conductive adhesive that can be cured at high temperature. The heat treatment used in such assembly process could cause degradation of piezoelectric properties in the PZT ceramics, subsequently affecting the performance of the devices [6-8]. The adhesive is usually cured at the range of $80-120^{\circ} \mathrm{C}$. Since the working temperature of piezoelectric ceramics cannot exceed $50 \%$ of Curie points 
$\left(T_{\mathrm{C}}\right)$, this curing process can accelerate the aging degradation of piezoelectric properties and the reduction in actuator performance $[7,8]$.

Aging degradation of piezoelectric ceramics depends on the material type, poling process, and heat treatment conditions [9, 10]. Aging behavior of piezoelectric ceramics can be quantified by a mathematical model based on a stretched exponential decay function [1114]. To date, few researches have investigated the effects of heat treatment temperature on the aging behavior of piezoelectric ceramics. This work focuses on the effects of heat treatment temperature on the aging degradation of soft and hard PZT ceramics. Heat treatments at different temperatures from 30 to $80 \%$ of $T_{\mathrm{C}}$ were applied to the PZT. The aging of piezoelectric coefficient $\left(d_{33}\right)$ and planar electromechanical coupling factor $\left(k_{p}\right)$ of the PZT were measured after heat treatment. The mathematical model based on a linear logarithmic stretched exponential function that reflects the polarization-defect dipole interaction during aging was used to describe the effects of temperature on the aging behavior. The results from this work will be useful for improving the manufacturing process of PZT actuators.

\section{Experiment}

Two types of $\mathrm{PbZr}_{0.52} \mathrm{Ti}_{0.48} \mathrm{O}_{3}(\mathrm{PZT})$ ceramics were used in this study. They were soft PZT that doped with $\mathrm{Nb}$ (PZT-5A type), and hard PZT that doped with Fe (PZT-4 type). Curie temperatures $\left(T_{\mathrm{C}}\right)$ of the soft and the hard ceramics are 360 and $325^{\circ} \mathrm{C}$, respectively. The ceramics were polished into a pellet shape with thickness of $1 \mathrm{~mm}$ and diameter of 10 $\mathrm{mm}$. The ceramics were annealed at $500^{\circ} \mathrm{C}$ for 2 hours to remove any residual stress due to the polishing process. Silver electrodes were painted on the parallel surfaces using a colloidal silver paste. A sample was submerged in silicone oil and poled under a DC electric field of 3 $\mathrm{kV} / \mathrm{mm}$, which is equivalent to 1.7 coercive field $\left(E_{c}\right)$ and $2 E_{c}$ of the soft and the hard ceramics, respectively, for $10 \mathrm{mins}$ at $100^{\circ} \mathrm{C}$. Piezoelectric coefficient $\left(d_{33}\right)$ and planar electromechanical coupling factor $\left(k_{p}\right)$ of poled samples were then measured 7 times with 
time interval of $24 \mathrm{hrs}$. After that, the ceramics were annealed at the temperatures of $30 \%$, $40 \%, 50 \%, 60 \%, 70 \%$ and $80 \%$ of the ceramics' $T_{\mathrm{C}}$ for $10 \mathrm{~min}$. After heat treatment, the $d_{33}$ and $k_{p}$ values of the ceramics were measured 7 times with time interval of $24 \mathrm{hrs}$. The measurements were continuously performed 4 times with time interval of 1 week. Six samples from each type of ceramics were tested to give averaged results on the measured values. The $d_{33}$ values of the samples were measured by a $d_{33}$ meter (APC product inc. S5865). Polarization-electric field $(P-E)$ hysteresis loops at different states were measured by a Sawyer-Tower circuit. In the $P-E$ measurement, the poled samples were placed into a sample holder, where the positive polarity of the samples was opposite to the positive field. X-ray diffraction (XRD) patterns were collected by X-ray diffraction technique (D2 PHASER, Bruker, USA). Microstructures of fracture surfaces were observed by a scanning electron microscope (JSM-6010V, JEOL, Japan). Electrical impedance was measured at the frequency range from 1.5 to $3 \mathrm{kHz}$ via an impedance/gain-phase analyzer (Solartron). The impedance was plotted as a function of frequency by a ZPlot-impedance software. The resonance $\left(f_{\mathrm{m}}\right)$ and the anti-resonance $\left(f_{\mathrm{n}}\right)$ frequencies, which are the frequencies of the minimum and the maximum impedances, respectively, were applied to calculate a $k_{p}$ value using the following equation [15],

$$
k_{p} \cong \sqrt{\left[\left(2.51\left(f_{n}-f_{m}\right) / f_{n}\right)-\left(\left(f_{n}-f_{m}\right) / f_{n}\right)^{2}\right]}
$$

\section{Results and discussion}

\subsection{Piezoelectric coefficient}

Piezoelectric coefficient $\left(d_{33}\right)$ values of the soft and hard PZT at the beginning of the aging process were measured to be 375 and $315 \mathrm{pC} / \mathrm{N}$ respectively, which can be used to normalize the subsequent measured $d_{33}$ values. Plots of the normalized $d_{33}$ values before and after heat treatments at various temperatures are shown in Fig. 1(a) and (b), respectively. For both ceramics, the normalized $d_{33}$ value measured before heat treatment (at aging time $<168$ 
hrs) decreased gradually with increasing aging time. At this period (aging time $<168 \mathrm{hrs}$ ), aging degradation of the soft ceramic was faster than that of the hard ceramic. The $d_{33}$ of both ceramics decreased rapidly after the heat treatment. The decrease in $d_{33}$ was greater when heat treated at higher temperature. At each heat treatment temperature, change in normalized $d_{33}$ of the soft PZT is greater than that of the hard PZT. The normalized $d_{33}$ measured after heat treatments (at aging time $\geq 192 \mathrm{hrs}$ ) of both ceramics decreased exponentially with aging time.

In order to determine the effects of heat treatment temperatures on the aging rates of both ceramics, time dependence of the normalized $d_{33}$ after heat treatments is expressed as a logarithmic function, as in the following equation [16],

$$
\text { aging rate }=\frac{1}{\log \left(t_{2}\right)-\log \left(t_{1}\right)}\left(\frac{p_{2}-p_{1}}{p_{1}}\right)
$$

where $t_{1}$ and $t_{2}$ are the numbers of hours just after heat treatment (192 hrs) and after an aging process (1344 hrs), respectively. $p_{1}$ and $p_{2}$ are the piezoelectric properties measured at $t_{1}$ and $t_{2}$, respectively. Aging rates of the ceramics are listed in Table 1. It shows that for both soft and hard ceramics, aging rate increases with increasing heat treatment temperature. Moreover, aging rate of the soft ceramic is faster than that of the hard ceramic.

In order to determine aging characteristics, time dependence of the normalized $d_{33}$ after heat treatments is fitted to an exponential law, as in the following equation $[11,13]$,

$$
p_{33}=p_{33_{\infty}}+p_{33_{1}} \exp \left[-(t / \tau)^{v}\right]
$$

where $p_{33_{\infty}}$ is the piezoelectric property which is time independent, therefore, the second term on the right-hand side of the equation is also time dependent. $t$ is the aging time. $\tau$ is the characteristic relaxation time, which relates to aging rate. $v$ is the stretching exponent. The fitting parameters of the ceramics are listed in Table 2. Relaxation time $\tau$ of the samples heated at higher temperatures are less than those sampels heated at lower temperature. At 
each heat treatment temperature, relaxation time of the soft ceramic is longer than that of the hard ceramic.

\subsection{Electromechanical coupling factor}

Planar electromechanical coupling factor $\left(k_{p}\right)$ values were normalized to the $k_{p}$ values measured at the beginning of the aging process. The aging behavior represented by the normalized $k_{p}$ values before and after heat treatments are shown in Fig. 2(a) and (b), respectively. For both ceramics, the normalized $k_{p}$ value degraded rapidly after heat treatment and gradually decreased with increasing aging time. The time dependences of the normalized $k_{p}$ after heat treatments (at aging time $\geq 192 \mathrm{hrs}$ ) were fitted to the logarithmic and the exponential functions as in Eq. (2) and (3), respectively. The fitting parameters are listed in Table 1 and 2. For both soft and hard PZT ceramics, the aging rate increased while the relaxation time decreased with increasing heat treatment temperature. At each heat treatment temperature, aging rate and relaxation time of the soft PZT are greater than that of the hard PZT.

\subsection{Discussion}

Theoretically, three major mechanisms have been proposed to explain the aging behavior of ferroelectric materials [17]. These mechanisms are: (1) defect dipole effect (or volume effect), (2) domain effect, (3) grain boundary interface effect. All these aging mechanisms were mostly dominated by oxygen vacancies formed during the high temperature process. In the case of volume effect, the defect dipole is formed by the different potential of the polarities between acceptor ions and oxygen vacancies. This defect dipole can interact with the lattice polarization in the domain. For domain effect, oxygen vacancies diffused toward and pinned domain walls. For gain boundary interface effect, oxygen vacancies further diffused toward grain boundaries. It is known that the concentration of oxygen vacancies in the soft PZT is lower than that in the hard one [18]. This leads to a lower 
number of defects contributing to aging of the soft PZT as compared to that of the hard PZT. Based on the effects of oxygen vacancies, therefore, the soft PZT could exhibit a greater aging degradation of piezoelectric properties as compared to the soft PZT. The aging model based on the response of oxygen vacancies at different scales, i.e. volume, domain and grain boundary effects, will be discussed.

The experimental result shows that the aging rate of both soft and hard PZT increases with increasing temperature in heat treatment. The aging rates after heat treatment of poled soft and hard PZT are significantly different. The aging process of poled ceramics could be dominated by the reorientation of poled domains. The different number of oxygen vacancy concentration is believed to be the main cause of the difference aging behaviors of soft and hard PZT. The interaction between domains and defects hinders the reorientation of poled domains. As a result, the soft PZT has a lower interaction between domains and defects due to the lower number of oxygen vacancies. Our proposed model, showing the orientation of domains and the arrangement of oxygen vacancies at low $(0.3 T c)$ and high $(0.8 T c)$ heat treatment temperatures, is schematically illustrated in Fig. 3. Pink and blue hexagons represent ferroelectric domains of the soft and the hard PZT, respectively. In each domain, the polarization direction is indicated by the arrow direction. A defect dipole is represented by a white ellipse of which direction is indicated by the direction of the arrow inside the ellipse. A yellow circle in which a plus is embedded represents an oxygen vacancy. In the initial state of aging process, oxygen vacancies are embedded inside domains and act as defect dipoles. The effect of defect dipoles on ferroelectric domains was reported by Ren $e t$ al. [19]. Defect dipoles usually align their polar directions in the direction of the domain at which they are implanted to minimize local electric and elastic energies [20]. At this state, the presence of oxygen vacancies as the volume effect dominates aging of the materials. Due to the hindering of those defect dipoles, domains could not be completely poled, in particular, 
the hard PZT. This makes piezoelectric properties at the initial state of aging of the hard PZT to be lower than that of the soft one. Moreover, the grain of hard PZT is observed to be smaller than the grain of the soft PZT, as shown in Fig. 4. SEM micrographs of fracture surfaces show that the mixed mode of intergranular and intragranular fractures was observed in both soft and hard PZT. Moreover, both ceramics showed well-packed grains with homogeneity in size. The average grain sizes of the soft and hard PZT were 3.5 and $1.0 \mu \mathrm{m}$, respectively. Smaller-grained ceramics contain a greater number of grain boundaries. Grain boundaries act as low dielectric materials screening ceramic grains from applied electric field. This leads to the harder switching of domains of the hard PZT as compared to the soft PZT with larger grains. Because the hindering of oxygen vacancies as defect dipoles, the domains could not reorient to the original directions, i.e. the directions before poling. This results in a greater loss of poled state and, subsequently, a greater aging rate of the soft PZT as compared to the hard PZT.

Piezoelectric properties of both ceramics decrease with heat treatment, in particular, at high heat treatment temperature. At a higher temperature, the number of reoriented domains is greater than that at lower temperature, contributing to a greater degradation of the properties. This could be due to the reduction in the barrier energy for domain switching at high temperature [21, 22], where the volume of switchable domains is enlarged and lead to the increase in in the number of domains that loses their poled state. Moreover, thermal energy can activate oxygen vacancies to diffuse toward domain walls. After heat treatment, the number of the reoriented domains and the oxygen vacancies moving toward domain walls increases with increasing aging time. Oxygen vacancies can move to grain boundaries to stabilize domains with further increasing aging time. These effects contribute to aging of ceramics. At the same heat treatment temperature, the properties degradation and the aging rate of the soft PZT are greater than that of the hard PZT. Because a lower concentration of 
oxygen vacancies, the soft PZT possesses a weaker interaction strength between domains and oxygen vacancies. This results in the greater number of reoriented domains and, subsequently, the greater properties degradation after heat treatment and aging rate. It can be seen that in all aging states, although the soft PZT shows the greater aging degradation and aging rate, however, the values of properties after aging are still higher than those of the hard PZT, as shown in Fig. 5. This implies that although the reorientation to the original direction of poled domains of the soft PZT is easier than that of the hard PZT, however, the number of remaining poled domains of the soft PZT is still greater than that of the hard PZT. For oxygen vacancies, besides they hinder domains to orient in poling process, moreover, they prevent the poled domains to reorient back to original directions as well. As compared to the soft PZT, a higher concentration of oxygen vacancies results in the lower aging degradation and aging rate of the hard PZT. However, due to the high concentration of oxygen vacancies, they cause the observation of pinched and asymmetric $P-E$ loops at before and after poling, respectively, as shown in Fig. 6. Moreover, the oxygen vacancies stabilized by diffusing toward domain walls and grain boundaries cause the shift of $P-E$ loop as well as the decrease in remanent polarization $\left(P_{r}\right)$ after heat treatment. For the soft PZT, besides a reduction of remanent polarization after heat treatment, such pinching and shifting of $P-E$ loops are not observed. In addition, a higher oxygen vacancy concentration also results in a greater change of $P-E$ loops after heat treatment. For example, the area inside the $P-E$ loops of the soft and the hard PZT reduces $14 \%$ and $17 \%$, respectively, after heat treatment. From the curve fitting of $d_{33}$ and $k_{p}$ (see Fig. 1 and 2), the relaxation time ( $\tau$ ) of soft and hard PZT decreases with increasing heat treatment temperature. This could be attributed to the decrease in the interaction strength between defect dipoles and dipole moments due to thermal energy provided by heat treatment $[23,24]$. 


\section{Conclusions}

In this work, the effects of heat treatment temperatures on aging behaviors of soft and hard PZT ceramics are investigated. For both ceramics, the degradation in the piezoelectric properties and the aging rate after heat treatment increase with increasing heat treatment temperature, while the relaxation time decreases. At each heat treatment temperature, the degradation of the piezoelectric properties after heat treatment, the aging rate and the relaxation time of the soft PZT are greater as compared to the hard PZT. These results show that heat treatment temperature attribute significantly to the aging degradation behaviors of the soft and the hard PZT ceramics. The difference in their aging behaviors could be associated with the concentration of oxygen vacancies in the ceramics. This work provides a model in explaining the effect of temperature on the aging degradation of soft and hard PZT. This model can be used to predict the aging behavior of PZT ceramics after heat treatment. It will be useful to prevent aging degradation of PZT ceramics from heat treatment processes.

\section{Acknowledgements}

This research has been supported by Suranaree University of Technology and the Higher Education Research Promotion and National Research University Project of Thailand, Office of the Higher Education Commission.

\section{References}

[1] F. S. Foster, L. K. Ryan, D. H. Tumbull, Characterization of lead zirconate titanate ceramics for use in miniature high-frequency $(20-8-\mathrm{MHz})$ transducers, IEEE Trans. Ultrason. Ferroelectr. Freq. Control. 38 (1991) 446-453. 
[2] G. Helke, S. Seifert, S.-J. Cho, Phenomenological and structural properties of piezoelectric ceramics based on $\mathrm{xPb}(\mathrm{Zr}, \mathrm{Ti}) \mathrm{O}_{3}-(1-\mathrm{x}) \mathrm{Sr}\left(\mathrm{K}_{0.25} \mathrm{Nb}_{0.75}\right) \mathrm{O}_{3}(\mathrm{PZT} / \mathrm{SKN})$ solid solutions, J. Eur. Ceram. Soc. 19 (1999) 1265-1268.

[3] Q.-M. Wang, Q. Zhang, B. Xu, R. Liu, L. E. Cross, Nonlinear piezoelectric behavior of ceramic bending mode actuators under strong electric fields, J. Appl. Phys. 86 (1999) $3352-3360$.

[4] J. Deng, W. Zhu, O.K. Tan, X. Yao, Amorphous $\mathrm{Pb}(\mathrm{Zr}, \mathrm{Ti}) \mathrm{O}_{3}$ thin film hydrogen gas sensor, Sens. Actuators, B 77 (2001) 416-420.

[5] Piezoelectric actuators and motors - Types, applications, new developments, industry structure and global markets.

http://www.innoresearch.net/report_summary.aspx?id=86\&pg=156\&rcd=ET$119 \& p d=10 / 1 / 2013($ accessed 1.03.16).

[6] M. Alguero, F. Guiu, M. J. Reece, Degradation of PZT-4D hard piezoceramics under moderate heating, J. Eur. Ceram. Soc. 20 (2000) 2705-2711.

[7] G.Xu, X. Wang, D. Yang, Z. Duan, C. Feng, Peculiar temperature aging effects on the piezoelectric constant of $\mathrm{Pb}\left(\mathrm{Mg}_{1 / 3} \mathrm{Nb}_{2 / 3}\right) \mathrm{O}_{3}-\mathrm{PbTiO}_{3}$ single crystal near the morphotropic phase boundary, Appl. Phys. Lett. 86 (2005) 032902/1-3.

[8] D. A. DeAngelis, D. C. Schalcosky, The effects of PZT8 piezoelectric crystal aging on mechanical and electrical resonances in ultrasonic transducers, IEEE Ultrason. Symp. (2006) 1935-1938.

[9] J. P. deMedeiros, M. H. Lente, J. A. Eiras, Influence of poling temperature on aging and thermal stability of PZT ceramics, IEEE Ultrason. Symp. 2 (1994) 955-958.

[10] A. Barzegar, R. Bagheri, A. K. Taheri, Aging of piezoelectric composite transducers, J. Appl. Phys. 89 (2001) 2322-2326. 
[11] Q. M. Zhang, J. Zhao, L. E. Cross, Aging of the dielectric and piezoelectric properties of relaxor ferroelectric lead magnesium niobate-lead titanate in the electric field biased state, J. Appl. Phys. 79 (1996) 3181-3187.

[12] S. Priya, J. Ryu, K. Uchino, D. Viehland, Mechanical aging behavior of oriented $\mathrm{Pb}\left(\mathrm{Mg}_{1 / 3} \mathrm{Nb}_{2 / 3}\right) \mathrm{O}_{3}-\mathrm{PbTiO}_{3}$ and $\mathrm{Pb}\left(\mathrm{Zn}_{1 / 3} \mathrm{Nb}_{2 / 3}\right) \mathrm{O}_{3}-\mathrm{PbTiO}_{3}$ single crystals, Appl. Phys. Lett. 79 (2001) 2624-2626.

[13] J. H. Koh, S. J. Jeong, M. S. Ha, J. S. Song, Aging of piezoelectric properties in $\mathrm{Pb}(\mathrm{MgNb}) \mathrm{O}_{3}-\mathrm{Pb}(\mathrm{ZrTi}) \mathrm{O}_{3}$ multilayer ceramic actuators, J. Appl. Phys. 96 (2004) $544-548$.

[14] D. Xue, J. Gao, L. Zhang, H. Bao, W. Liu, C. Zhou, X. Ren, Aging effect in paraelectric state of ferroelectrics: Implication for a microscopic explanation of ferroelectric deaging, Appl. Phys. Lett. 94 (2009) 082902/1-3.

[15] APC International Ltd., Piezoelectric Ceramics: Principles and Applications, USA: Mackeyville; 1900.

[16] D. J. Jones, S. E. Prasad, J. B. Wallace, Piezoelectric materials and their applications, Key Eng. Mater. 122-124 (1996) 71-144.

[17] K. Carl, K. H. Härdtl, Electrical after-effects in $\mathrm{Pb}(\mathrm{Ti}, \mathrm{Zr}) \mathrm{O}_{3}$ ceramics, Ferroelectrics 17 (1978) 473-486.

[18] G. H. Haertling, Ferroelectric ceramics: History and technology, J. Am. Ceram. Soc. 82 (1999) 797-818.

[19] X. Ren, Large electric-field-induced strain in ferroelectric crystals by point-defectmediated reversible domain switching, Nat. Mater. 3 (2004) 91-94.

[20] P. Erhart. P. Träskelin, K. Albe, Formation and switching of defect dipoles in acceptordoped lead titanate: A kinetic model based on first-principles calculations, Phys. Rev. B 88 (2013) 024107/1-10. 
[21] W. Pan, C. F. Yue, O. Tosyali, Fatigue of ferroelectric polarization and the electric field induced strain in lead lanthanum zirconate titanate ceramics, J. Am. Ceram. Soc. 75 (1992) 1534-1540.

[22] C. Brennan, Model of ferroelectric fatigue due to defect/domain interactions, Ferroelectrics 150 (1993) 199-208.

[23] M. I. Morozov, D. Damjanovic, Charge migration in $\mathrm{Pb}(\mathrm{Zr}, \mathrm{Ti}) \mathrm{O}_{3}$ ceramics and its relation to ageing, hardening, and softening, J. Appl. Phys. 107 (2010) 034106/1-10.

[24] S. Gottschalk, H. Hahn, S. Flege, A. G. Balogh, Oxygen vacancy kinetics in ferroelectric $\mathrm{PbZr}_{0.4} \mathrm{Ti}_{0.6} \mathrm{O}_{3}$, J. Appl. Phys. 104 (2008) 114106/1-7. 


\section{Figure captions}

Fig. 1 The time dependence of normalized $d_{33}$ values before and after heat treatments at various temperatures of (a) the soft and (b) the hard PZT ceramics.

Fig. 2 The time dependence of normalized $k_{p}$ values before and after heat treatments at various temperatures of (a) the soft and (b) the hard PZT ceramics.

Fig. 3 The schematical model showing the orientations of domains and oxygen vacancies as a function of aging time before and after heat treatments at (a) low and (b) high temperatures of the soft and the hard PZT ceramics. The pink and blue hexagons represent the ferroelectric domains of the soft and the hard ceramics, respectively. The arrows embedded in the domains represent the affective directions of the polarizations inside the domains. The ellipses represent the defect dipoles. The defect dipoles' directions are demonstrated by the directions of the arrows embedded the ellipses. A yellow circle in which a plus is embedded represents an oxygen vacancy. The light-pink and the light-bule regions indicate the depolarizations.

Fig. 4 SEM images of fracture surfaces of (a) the soft and (b) the hard PZT ceramics.

Fig. 5 The time dependence of real values of $d_{33}$ before and after heat treatments at various temperatures of (a) the soft and (b) the hard PZT ceramics.

Fig. 6 The $P-E$ hysteresis loops measured at differernt states of (a) the soft and (b) the hard PZT ceramics. 


\section{Table captions}

Table 1 Aging rates calculated from the time dependence of normalized $d_{33}$ and $k_{p}$ at various heat treatment temperatures of soft and hard PZT ceramics.

Table 2 Relaxation times obtained from fitting time dependence of normalized $d_{33}$ and $k_{p}$ at various heat treatment temperatures of soft and hard PZT ceramics. 
Fig. 1
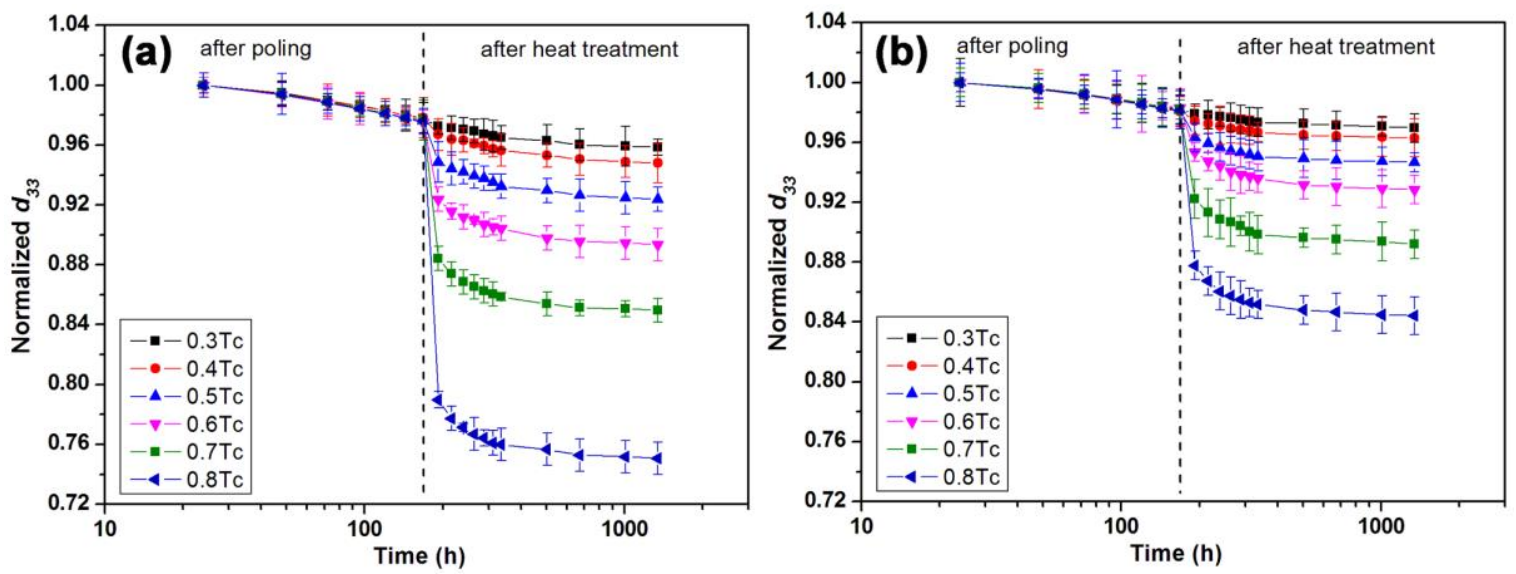

Fig. 2
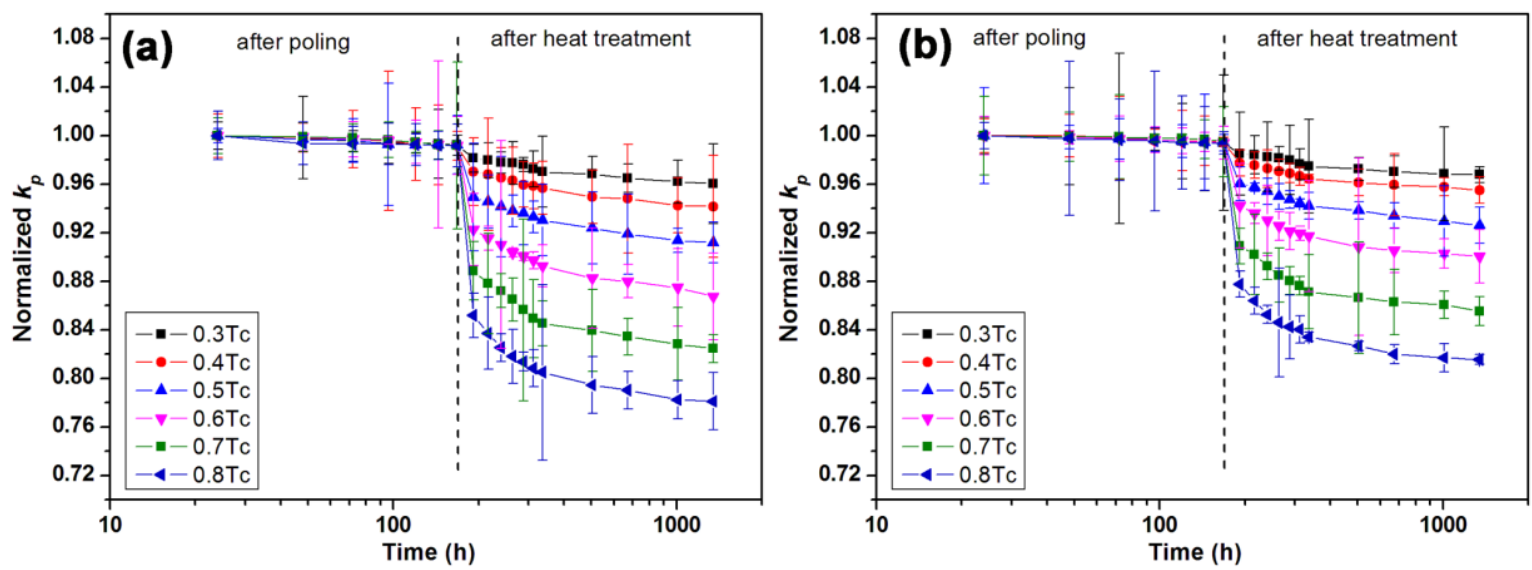

Fig. 3

(a)

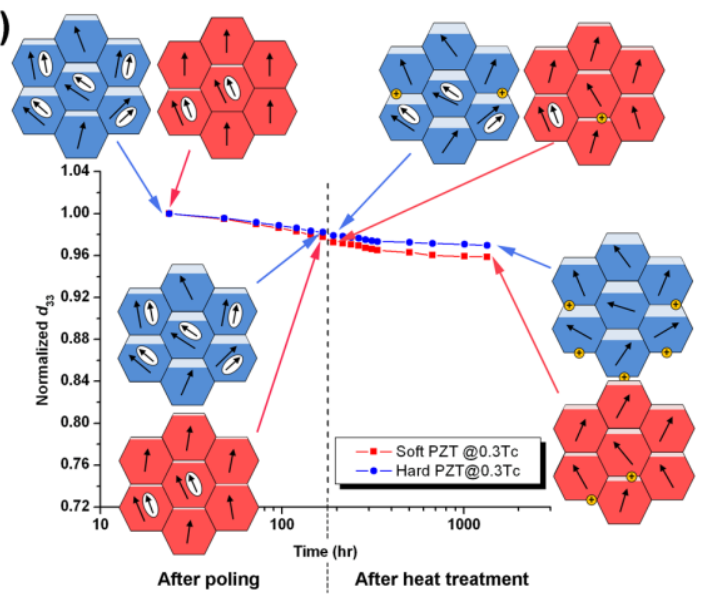

(b)

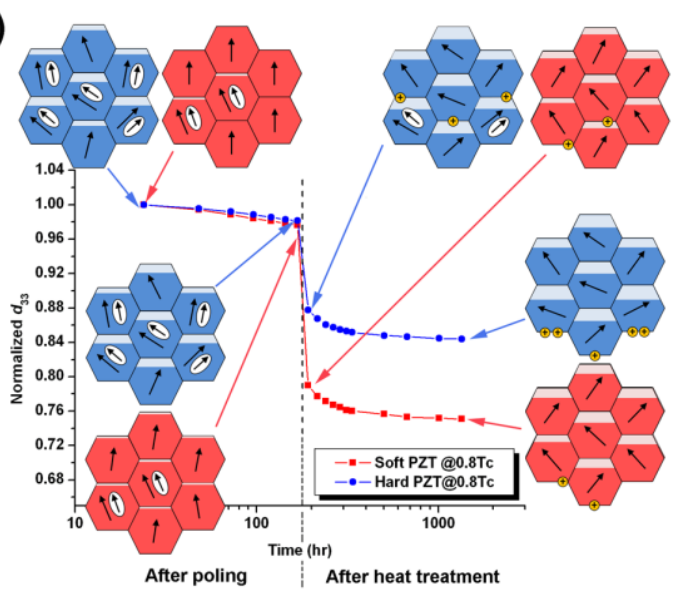


Fig. 4
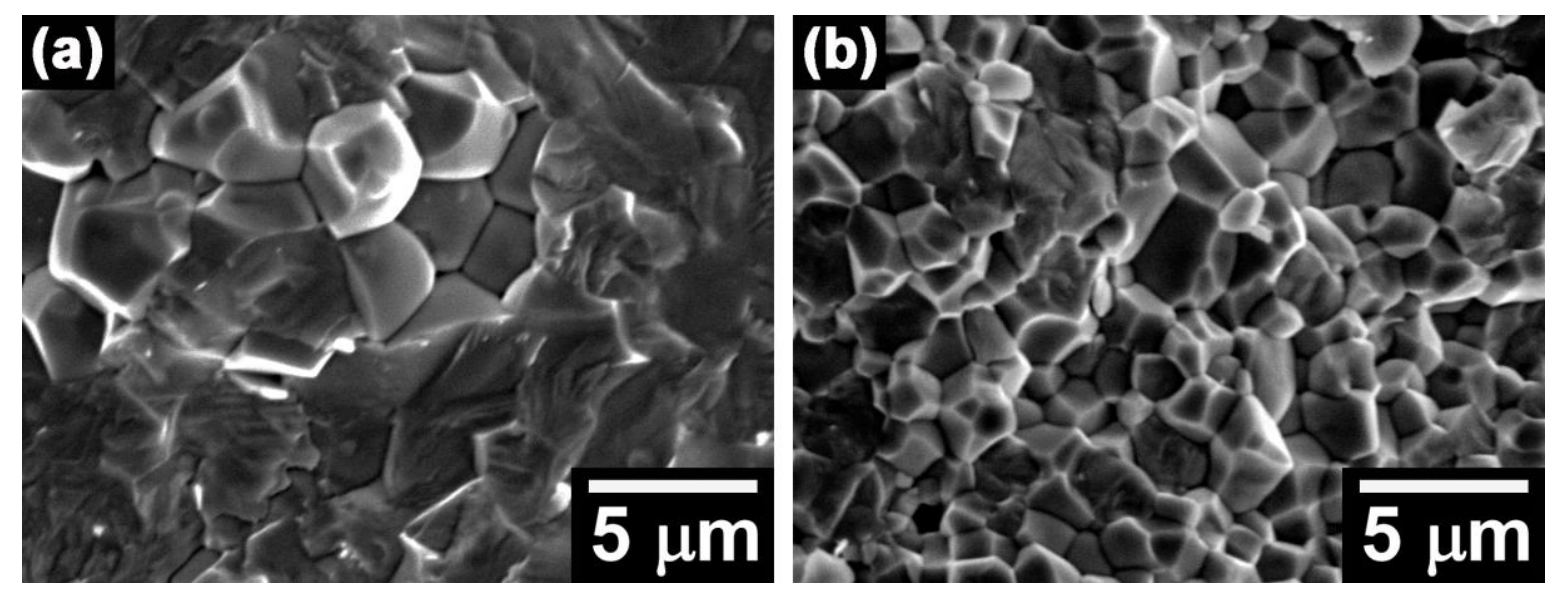

Fig. 5
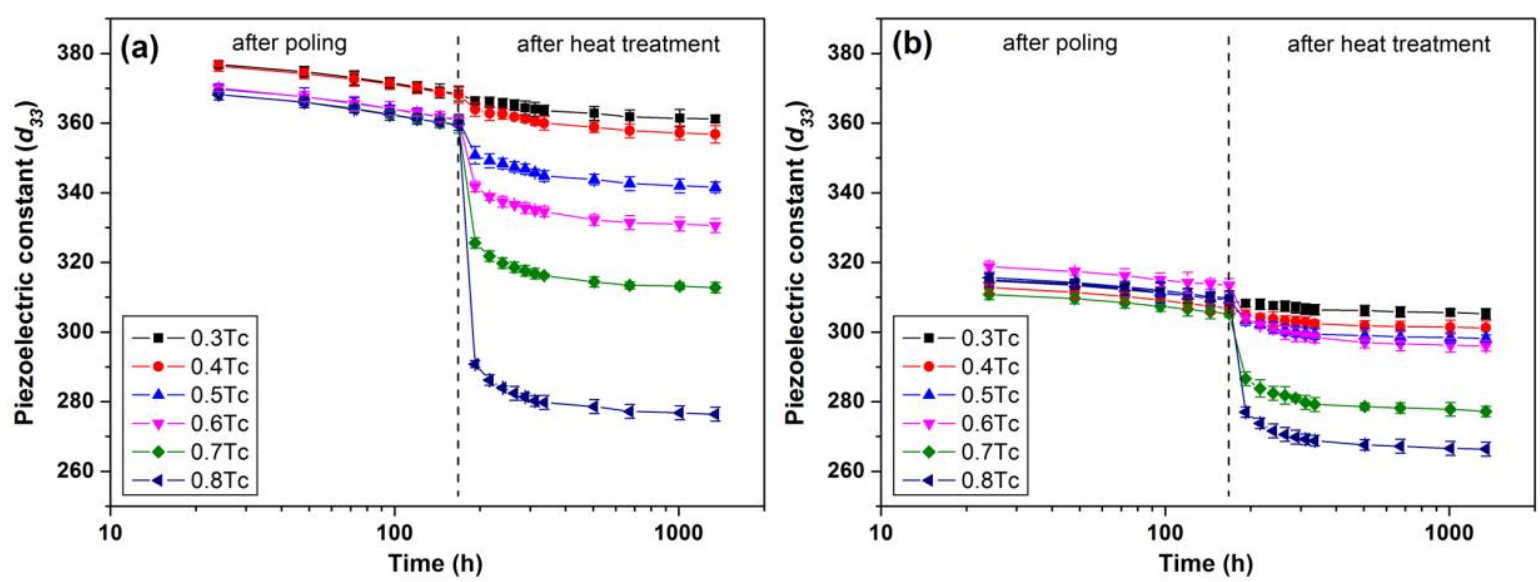

Fig. 6

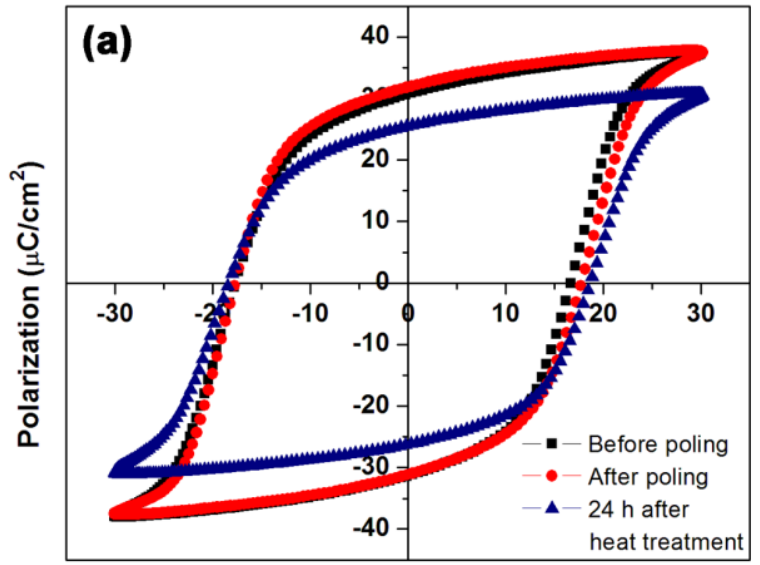

Electric field $(\mathrm{kV} / \mathrm{cm})$

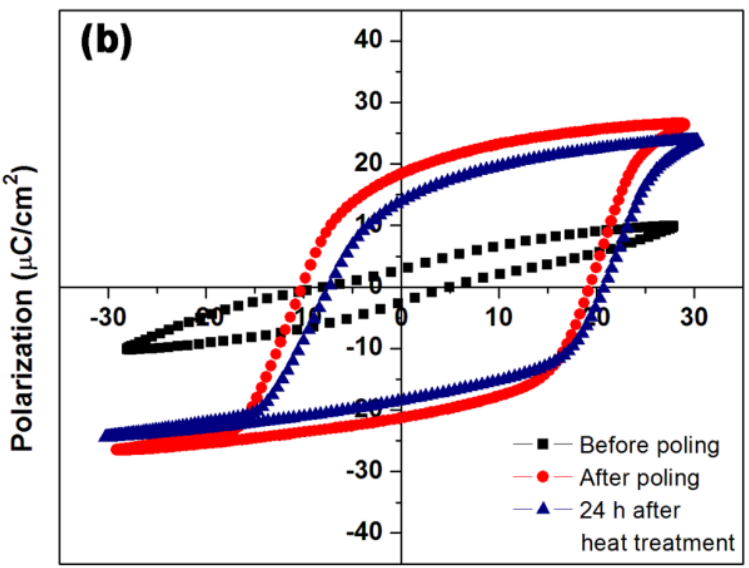

Electric field $(\mathrm{kV} / \mathrm{cm})$ 
Table 1

\begin{tabular}{ccccc}
\hline Heat treatment & \multicolumn{2}{c}{ Aging rate from $d_{33}$ fitting } & \multicolumn{2}{c}{ Aging rate from $k_{p}$ fitting } \\
\cline { 2 - 5 } temperature $\left(\% T_{C}\right)$ & Soft PZT & Hard PZT & Soft PZT & Hard PZT \\
\hline 30 & 0.0168 & 0.0115 & 0.0254 & 0.0205 \\
40 & 0.0234 & 0.0140 & 0.0349 & 0.0269 \\
50 & 0.0310 & 0.0203 & 0.0465 & 0.0422 \\
60 & 0.0388 & 0.0311 & 0.0708 & 0.0523 \\
70 & 0.0465 & 0.0388 & 0.0856 & 0.0700 \\
80 & 0.0586 & 0.0453 & 0.0982 & 0.0839 \\
\hline
\end{tabular}

Table 2

\begin{tabular}{ccccc}
\hline \multirow{2}{*}{$\begin{array}{c}\text { Heat treatment } \\
\text { temperature }\left(\% T_{C}\right)\end{array}$} & \multicolumn{2}{c}{$\tau$ from $d_{33}$ fitting $(\mathrm{hr})$} & \multicolumn{2}{c}{$\tau$ from $k_{p}$ fitting $(\mathrm{hr})$} \\
\cline { 2 - 5 } & Soft PZT & Hard PZT & Soft PZT & Hard PZT \\
\hline 30 & 213 & 178 & 331 & 237 \\
40 & 176 & 115 & 231 & 190 \\
50 & 126 & 76 & 212 & 137 \\
60 & 76 & 64 & 130 & 117 \\
70 & 31 & 34 & 100 & 82 \\
80 & 22 & 18 & 54 & 57 \\
\hline
\end{tabular}

